\title{
Endoscopic Spinal Decompression from an Interventional Spine Physician's Perspective
}

\section{Bernard MK Lee}

Department of Pain Management, Singapore Pain care Center, Singapore

*Corresponding author: Bernard MK Lee, Director, Department of Pain Management, Singapore Pain care Center, Singapore, Tel: + 6598000499; E-mail: drblee@paincarecenter.com.sg

Rec Date: August 06, 2018; Acc Date: August 26, 2018; Pub Date: September 4, 2018

Copyright: () 2018 Lee BMK. This is an open-access article distributed under the terms of the Creative Commons Attribution License, which permits unrestricted use, distribution, and reproduction in any medium, provided the original author and source are credited.

\begin{abstract}
Mechanical low back pain conditions have been traditionally treated with open surgical spinal decompression. The approach to the spine has evolved towards using minimally invasive techniques, previously microdiscectomy and now endoscopic spine surgery (ESS). ESS has bridged the gap between the frustrations of conservative measures with opioid medications to open surgery with inherent surgical and anaesthetic risks. ESS offers a direct aware state means of localizing and treating neuro-claudicant back pain, referred pain and weakness associated with stenosis that fail to respond to rehabilitation, pain management or surgery. In this letter to the editor, I discuss the endoscopic spinal decompression from an interventional spine in view of a physician's perspective.
\end{abstract}

Keywords: Low back pain; Spine decompression; Disc pathology; Spine surgery; Post-laminectomy syndrome

\section{Introduction}

I refer to the article by Yeung, and co-authors on the suggestions for a practical and progressive approach to endoscopic spine surgery training and privileges [1,2]. "Endoscopic spine surgery blends skill sets, technology, and clinical application from both surgical spine and interventional spine". I concur with Yeung's views that "the lines between surgical and nonsurgical treatment are blurred by technology and evolving practice patterns. Some interventional spine practitioners are able to provide safe and effective endoscopic spine surgery, but the training and practice standards have to be established." Furthermore, the learning curve for specific ESS procedures may also need to be defined to ensure patient safety $[2,3]$.

Despite having been fully dedicated as an Interventional Spine Physician (ISP) of many years, performing many spinal procedures ranging from percutaneous disc ablation, disc nucleotomy, radiofrequency medial branch neurotomy, implantation of spinal cord stimulators, intra thecal pumps, dorsal root ganglion modulation; venturing into ESS was not an easy task - both clinically and administratively. Before embarking on the ESS journey, one should have adequate experience in disc ablation treatment [4]. Being able to handle disc pathology, starting from smaller needles (Nucleoplasty/ IDET needles) and progressing to larger cannulas such as Disc FX, allowed me to progress to minimally invasive spine decompression. Having gone through hundreds of Nucleoplasty (Coblation using radiofrequency vaporization of nuclear tissue) and percutaneous disc decompression (Dekompressor quantifiable extraction of disc material to achieve volume reduction), there remains many patients with a sizeable disc herniation having mechanical nerve root compression that would benefit from a more directed approach to decompress the affected nerves.

\section{Endoscopic Journey as an ISP}

My ISP journey started with the teachings and mentoring from Anthony Yeung. His "inside out" YESS (Yeung Endoscopic Spine System) transforaminal approach, showed that disc decompression using fluoroscopically guided percutaneous techniques, is an easier, safer, readily reproducible, and more cost-effective treatment [5]. It creates less surgical morbidity than traditional open surgical methods [5]. Having a good grasp of the radio-anatomy of the spine with reference to the underlying structures (e.g. medial pedicular line, posterior vertebral line and spinal canal shadow) rapidly advanced my learning and competency. Placement of the guide wire within the targeted disc area was an extension of Disc FX technique. The "inside out" approach gave me confidence during the initial handling of endoscopic equipment. Over time, the progression towards an "outside-in" approach (pioneered by Thomas Hoogland via the Thessys by Joimax) was more helpful in decompression of larger disc herniation and visualisation of the transversing nerve root. Learning and applying principles from Ruetten to decompress canal stenosis and lateral recess stenosis via translaminar endoscopic technique was an extension from interlaminar disc decompression of L5/S1 (especially patients with difficult access to L5/S1 via the transforaminal approach) [6].

Can interventional pain practitioner learn and be competent in ESS? My humble opinion would be with adequate training and exposure, an ISP can be trained adequately. The approach to disc ablation via nucleoplasty, IDET and Disc FX has been delivered professionally within the perview of ISP. These procedures approached the disc via the transforaminal access. Traditional spine surgeons have not historically embraced the field, leading to ISP starting to fill the vacuum. Many of the skills developed during training as an ISP translate directly to the practice of ESS [2]. The size of the endoscopic cannulas ranging from $7-9 \mathrm{~mm}$ OD is bigger than that of Disc FX. As ISP starts on this learning process, one would need to acknowledge the limitations of foraminal access and have an adequate knowledge of the Kambin's triangle and its boundaries, to allow for safe delivery of this technique. Bone preservation and judicious dissection using less 
traumatic instrument under local anaesthesia coverage would be the initial experience of the ISP, until one is used to looking at the tissue structures via the scope. To minimize complications including failed back surgery syndrome, one should carefully select suitable patients and start with less complex patients' spinal pathology. Endoscopic techniques can vary and will likely depend on the "surgeon factor" in obtaining favourable results [7]. The ability to evaluate the area in and around the foramen and nerves opens a whole new dimension for the evaluation and treatment of common and complex back pain [7]; ISP has been contributing to this additional dimension of providing pain relief in refractory pain following post-laminectomy syndrome.

Surgical risk such as nerve injury, bleeding, dura tear and infection should be thoroughly discussed with patients especially for procedures such as epidurolysis, SELD (trans-sacral epiduroscopic lumbar decompression, spinal cord stimulator, intrathecal pump implantation and disc ablation. The management of the risk in ESS is no different from those mentioned above. Patients need to be informed about the possibility of a staged surgery in case of complication. The need for a surgical back up cannot be overstated; this arrangement mirrors that of cardiothoracic surgeon being a backup for interventional cardiologist in percutaneous coronary angioplasty.

Training via hands-on cadaveric workshops has been invaluable for me to develop safe approaches in ESS [7]. Training under different endoscopists, who have their own favoured style and technique to overcome possible obstacles, were important in my learning journey. I had to invest additional training in understanding principles of surgical spine care, complication recognition and management and the biomechanics of the spine [2], to bridge the gaps in my training of spine care. Having gained experience and interacting with the "best" in the field, I was able to share my limited experience at various minimally invasive spine conferences (Asean and Asian MISST) in my region.

\section{Case Series}

The following case series of 51 patients underwent endoscopic lumbar spine decompression from July 2016 to May 2018, constituting of 16 cases of transforaminal lumbar disc decompression (L3/L4 - L4/ L5), 10 cases of interlaminar lumbar disc decompression (L5/S1) and 25 cases of interlaminar spinal stenosis decompression (L4/L5 or L5/ S1). Of the 51 patients, 27 were female and 24 were male with the average age of 50 years old for disc decompression and 70 years old for canal stenosis decompression. All patients reported VAS $>5 / 10$. The Oswestry disability scores were $>45 \%$. They have low back pain and radiculopathy without any neurological deficit. They reported claudication distance of 20 minutes and less. For disc herniation cases, their straight leg raise of the affected limb was less than 60 degrees. Their MRI documentation showed disc protrusion of at least Grade 2B MSU classification of disc herniation [8]. Patients with high migration of disc extrusion were excluded. For patients suffering from spinal canal stenosis due to facet hypertrophy and thickened ligamentum flavum, their MRI lumbar spine showed effacement of CSF but cauda equine rootlets may or may not be recognizable, or dural sac being homogenous gray with no CSF signal visible. The lateral recess angle of the affected side was less than 10 degrees.

Preparation of the patients included explaining that traditional open surgical decompression was the mainstay of treatment. This alternative approach via endoscopic assisted spinal decompression was performed by myself as an Interventional Spinal Physician. Should there be any need, a follow on open surgical laminectomy decompression by a neurosurgeon may be necessary. Those scenarios explicitly stated included inadequate decompression, uncontrollable bleeding and nerve injury +/- dura tear. Patients were selected for single level decompression despite multi-level disease. The primary end-point was pain relief and not reversal of degenerative disease. Patients with unstable spine of more than grade 1 spondylolisthesis were excluded from this treatment.

All patients were performed under moderate sedation. They were monitored overnight and discharged the next day. They were given a basic back corset brace to be worn for 1 month during daytime.

All the cases were performed using the Joimax endoscopic system. For disc herniation cases, transforaminal approach was done for L3/L4 - L4/L5 levels using the TESSYS $7 \mathrm{~mm}$ OD (outer diameter). For the L5/S1 disc herniation, interlaminar disc decompression using iLESSYS $7 \mathrm{~mm}$ OD was chosen due to high riding iliac crest. For canal stenosis cases, ipsilateral and contra-lateral decompression of the lateral recess and spinal canal were achieved using the iLESSYS $7 \mathrm{~mm}$ OD. Bony decompression of the lamina and facet were performed using a diamond bur Shrill Shaver.

For transforminal disc decompression, the visualization of the freely mobile dura and the transversing root marks adequate decompression. For adequacy of interlaminar canal stenosis decompression it was the ability to stretch the exiting root side to side. After the decompression, radiofrequency ablation of the respective medial branches of facet joints and nerve root block were done.

\section{Results}

The mean pre-operative VAS scores were 7.7 while the postoperative VAS scores at POD1 were 2.3 and 1.9 at 1 month review. For those disc herniation cases, the improvement of straight leg-raise to 90 degrees was evident at POD1. These values indicate a significant improvement in patients' back pain and radiculopathy. Their average walking tolerance has increased by $77.6 \%$ and their satisfaction scores at 4.1 out of 5 .

The persistence and recurrence of patient's radicular pain is around $10 \%$. Two patients from the transforaminal disc decompression and three patients from the interlaminar canal stenosis decompression group. One patient with L4/L5 grade $2 \mathrm{~B}$ disc herniation developed recurrence of pain by $2^{\text {nd }}$ week. The preop VAS $10 / 10$ and post op POD1 VAS $3 / 10$. By $2^{\text {nd }}$ week, the VAS $5 / 10$. The straight leg raise improved to $90 / 90$ from $40 / 90$. Repeat MRI showed inadequate decompression at the superior vertebral notch. Eventually, the patient underwent open laminectomy decompression at $4^{\text {th }}$ week. Feedback from neurosurgeon revealed a small hardened disc fragment lodged at the notch during open surgery.

One patient had recurrence of pain at 6 months post procedure associated with a previous surgery with discectomy L4/L5 and DIAM space implant 4 years prior. There was back pain with radiculopathy from a L4/L5 grade $3 \mathrm{~A}$ disc herniation. The preoperative VAS 8/10, postoperative VAS $2 / 10$. Post procedure the straight leg raise was $80 / 80$ from $40 / 70$ and there was adequate pain relief for 5 months. Repeat MRI showed recurrence of disc herniation, likely to be compounded by the DIAM spacer. The patient underwent laminectomy decompression and fusion subsequently.

Two patients had persisting pain at one-month review - inadequate decompression of canal stenosis. These were early cases in the learning 
Page 3 of 3

curve of not achieving adequate decompression of lateral canal stenosis with endoscopic interlaminar stenosis decompression. One particular patient had persistent pain due to inability to decompress the spinal canal from equipment failure. He had severe L4/L5 canal stenosis, severe narrowing at the lateral recess angle and effacement of CSF space with no recognizable nerve rootlets. During the interlaminar endoscopic decompression, the diamond bur Shrill Shaver malfunctioned. There was no back up shaver available. The stenotic canal could not be adequately decompressed due to the lack of correct instrument. The tight space between upper and lower lamina made it difficult to visualize structures. Attempts to decompress the lamina and lavum were made with Kerrison Punch and Rongeur forceps. Due to the sub-optimal working space for the scope, an inadvertent dura tear was made. The patient was treated conservatively initially for the asymptomatic dura tear (2\%). There were no low CSF pressures signs nor any meningitis issues. The decision to adopt an expectant management regarding the dura tear was made between patient and Neurosurgeon. However, he was disabled by the persistent back pain from the lumbar canal stenosis. Three weeks later, he underwent open laminectomy decompression and fusion.

\section{Discussion}

It is imperative that backup instruments be available in case of any equipment failure. It was a difficult choice to make whether to abandon the procedure when the shaver failed. It may be perceived to be more acceptable to have a "failed back surgery" outcome than to have an asymptomatic dura tear. Recognizing the lack of equipment, and needing a neurosurgical input might have been the Hobson's choice, hence planning it as a staged procedure. While dura tear is an accepted surgical risk, for the interventional spine physician, the treatment of this inadvertent outcome becomes a torching point, even if we could attempt to seal the tear with a blood patch or Fibrin Sealant patch. This $2 \%$ incidence of dura tear is arguably a modest comparison to the $1 \%$ to 7\% reported for microdiscectomy surgeries [9]. Complication management has to be managed early in the pre-procedure planning phase. Extensive discussion is necessary to calibrate patient's expectation and informed consent of these risks. While ISP undergoes training and learn the necessary anatomical knowledge, relevant skill set and selection of patients, the need to work closely within a multidisciplinary set up prevails. Open surgical interventions could be a consideration for patients in a staged management plan. Similar collaborations between cardiothoracic surgeon intervening for interventional cardiologist and ISP being involved in the care of failed back surgery syndrome patients established the mutual need for interdiscipline care.

Persistent pain a ter spine surgery varies between $1 \%$ to $20 \%$ [10] The $10 \%$ recurrence of pain in this case series is matched modestly within expectation. As an ISP, the ability to offer a minimally invasive procedure such as ESS to patients, ills the void needed to decompress the spine mechanically. I would expect the incidence of persistent pain to reduce over time.

To address the lack of formalized and peer reviewed training programs for evaluation of surgical skill and judgment, while selfevaluation is our moral compass, ISP needs to incorporate a morbidity regular peer review learning mechanism though it is not dedicated to just ESS. Efforts are made to ensure that performance standards are upheld adequately, such as starting our own cadaveric workshop.

\section{Conclusion}

As ISPs incorporate endoscopic spine surgery in our field of specialization, we must have adequate training and background experience. This is best practiced in a multi-disciplinary environment, together with neurosurgeon and orthopaedic surgeon alike. Each stakeholder should evolve by working together rather than be concerned about "protecting their turf" and using all the individual skills for treating their mutual patients [7]. The political sensitivity regarding ISP overstepping into orthopaedic territory regarding ESS should be set aside as in the example of China's ISP embracing ESS [7]. Tracheostomy is a good example of various stakeholders working together for the best interest of the patient. ENT surgeon, intensivist (anaesthesiologist), respiratory physicians all can perform Tracheostomy. Within my own work, there are surgical colleagues acknowledge and support me as an ISP performing ESS, while others could be less accepting. I look forward to the synergistic collaboration amongst all stakeholders, especially in the area of training and accreditation, for the progress and evolution of ESS, especially taking the examples from China and Korea.

\section{References}

1. Yeung AT, Yeung CA (2007) Minimally invasive techniques for the management of lumbar disc herniation. Orthop Clin North Am 38: 363-372.

2. Yeung AT, Roberts A, Shin P, Rivers E, Paterson A (2018) Suggestions for a practical and progressive approach to endoscopic spine surgery training and privileges. J Spine 7: 414.

3. Morgenstern R, Morgenstern C, Yeung AT (2007) The learning curve in foraminal endoscopic discectomy: An experience needed to achieve a $90 \%$ success rate. SAS J 1:100-107.

4. Yeung AT, Yeung CA, Salari N, Field J, Navratil J, et al. (2017) Lessons learned using local anesthesia for minimally invasive endoscopic spine surgery. J Spine 6: 377.

5. Yeung AT, Gore SR (2001) Evolving methodology in treating discogenic back pain by Selective Endoscopic Discectomy (SED) and thermal annuloplasty. J Minim Invasive Spine Surg Tech 1: 8-16.

6. Ruetten S, Komp M, Merk H, Godolias G (2008) Full-endoscopic interlaminar and transforaminal lumbar discectomy versus conventional microsurgical technique: A prospective, randomized, controlled study. Spine (Phila Pa 1976) 33: 931-939.

7. Yeung AT, Yeung CA, Salari N, Field J (2017) Endoscopic surgery of the spine as a multi-disciplinary subspecialty for appropriately trained, and experienced providers. J Spine 6: 388.

8. Mysliwiec LW, Cholewicki J, Winkelpleck MD, Eis GP (2010) MSU classification for herniated lumbar discs on MRI: Toward developing objective criteria for surgical selection. Eur Spine J 19:1087-1093.

9. Kalevski S, Peev N, Haritonov D (2010) Incidental dural tears in lumbar decompressive surgery: Incidence, causes, treatment, results. Asian J Neurosurg 5: 54-59.

10. Wera GD, Marcus RE, Ghanayem AJ, Bohlman HH (2008) Failure within one year following a subtotal lumbar discectomy. J Bone Joint Surg Am 90: 10-15.

This article was originally published in a special issue, Minimally Invasive Spine Surgery -Il handled by Editor(s). Anthony T. Yeung, M.D. Clinical professor, University of New Mexico School of Medicine Associate Desert Institute for Spine Care, Phoenix, Arizona, Executive director International Intradiscal therapy Society Phoenix, Arizona 